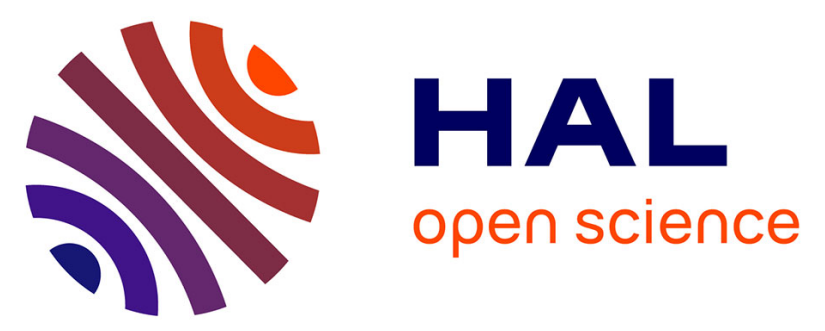

\title{
Activité peroxydasique et rhizogenèse dans le cas de Cynara scolymus L.: évolution au cours de repiquages successifs de boutures cultivées in vitro. Comparaison avec de jeunes plantes issues de graines \\ Charles Moncousin, Georges Ducreux
}

\section{To cite this version:}

Charles Moncousin, Georges Ducreux. Activité peroxydasique et rhizogenèse dans le cas de Cynara scolymus L.: évolution au cours de repiquages successifs de boutures cultivées in vitro. Comparaison avec de jeunes plantes issues de graines. Agronomie, 1984, 4 (2), pp.105-111. hal-00884617

\section{HAL Id: hal-00884617 https://hal.science/hal-00884617}

Submitted on 1 Jan 1984

HAL is a multi-disciplinary open access archive for the deposit and dissemination of scientific research documents, whether they are published or not. The documents may come from teaching and research institutions in France or abroad, or from public or private research centers.
L'archive ouverte pluridisciplinaire HAL, est destinée au dépôt et à la diffusion de documents scientifiques de niveau recherche, publiés ou non, émanant des établissements d'enseignement et de recherche français ou étrangers, des laboratoires publics ou privés. 


\title{
Activité peroxydasique et rhizogenèse dans le cas de Cynara scolymus L. : évolution au cours de repiquages successifs de boutures cultivées in vitro. Comparaison avec de jeunes plantes issues de graines
}

\author{
Charles MONCOUSIN \& Georges DUCREUX (*) \\ Ecole d'ingénieurs horticoles, CH 1254 Lullier (Suisse) \\ (*) C.N.R.S., Laboratoire d'Étude et d'Exploitation du Polymorphisme végétal, Université de Paris-Sud, \\ F 91405 Orsay Cedex
}

RÉSUMÉ

\begin{abstract}
L'analyse des aptitudes morphogénétiques de boutures-rosettes d'artichaut (Cynara scolymus L.) au fil de la multiplication végétative in vitro permet de montrer une réacquisition progressive de propriétés proches de celles de boutures-rosettes obtenues sur des plantes issues de graines. Les réponses aux tests d'enracinement et de callogenèse montrent une sensibilisation progressive des fragments aux divers traitements rhizogènes. L'adjonction d'ergocalciférol (vitamine $\mathrm{D}_{2}$ ) au meilleur traitement auxinique permet de stimuler les réponses rhizogènes tout en diminuant l'aptitude callogène.

L'utilisation de l'évolution de l'activité peroxydasique, comme marqueur, se révèle particulièrement adéquate pour apprécier les potentialités des boutures-rosettes soumises au traitement rhizogène optimal. Les réponses aux tests morphogénétiques et l'analyse de l'évolution de l'activité peroxydasique permettent de montrer, avec certitude, qu'il y a retour vers un fonctionnement du type de celui de la jeune plante issue de la graine au fil des cultures successives réalisées in vitro.
\end{abstract}

Mots clés additionnels: Multiplication conforme, rajeunissement, peroxydases, ergocalciférol, acide naphtalène acétique.

Peroxidase activity and rhizogenesis in Cynara scolymus L. : changes during in vitro subculture and comparison with young plants from seed.

\begin{abstract}
Analysis of the morphogenetic aptitudes of rosette cuttings of artichoke (Cynara scolymus $\mathbf{L}$.) during in vitro vegetative multiplication has shown progressive reacquisition of propertics close to those of rosette cuttings obtained from plants arising from secds. The responses to the rooting and callogenic tests showed progressive sensitization of the fragments to various rhizogenic treatments. The addition of ergocalciferol $\left(V i t a m i n D_{2}\right)$ to the best auxin treatment led to stimulation of the rhizogenic responses while decreasing callogenesis.

Peroxidase activity was particularly useful as a marker to assess the potentialities of rosette cuttings undergoing the optimal rhizogenic treatment. The morphogenetic tests and the peroxidase analyses showed, with a high degree of certainty, that there is a return, during succcssive cultures in vitro, to a mode of functioning similar to that of the young plant arising from seed.
\end{abstract}

Additional key words : Conformable multiplication, rejuvenation, peroxidase, ergocalciferol, naphtalene acetic acid.

\section{INTRODUCTION}

Depuis la jeune plante issue de la graine et qu'il est à l'évidence nécessaire d'appeler « jeune », le déploiement morphogénétique d'un végétal est caractérisé par un ensemble de phénomènes typiques affectant tant l'appareil aérien que l'appareil racinaire (NOZERAN \& BANCILHON, 1972 ; NOZERAN, 1978).
D'après DoOREnbos (1965), la phase de jeunesse serait caractérisée, outre ses particularités morphologiques, par sa grande potentialité à former des racines adventives. La diminution partielle ou totale de l'aptitude à l'enracinement adventif lors du passage de la phase de jeunesse à la phase ultérieure a d'ailleurs été notée par plusieurs auteurs (GREGORY, 1951 ; DOORENBOS, 1953 ; FRANCLET, 1969). Cependant la possibilité d'un retour au système initial de 
fonctionnement persiste au niveau de certaines zones de la plante (NOZERAN, 1978) et semble favorisé par l'emploi des cultures in vitro. Récemment, tentant de saisir le déterminisme de ces faits, NozERAN et al. (1982) ont pu avancer, en particulier, que le phénomène de rajeunissement - c'est-àdire, le retour à des structures et propriétés manifestées par la jeune plante issue de graine - pouvait être relić à 3 groupes de phénomènes capables d'intervenir concurremment ou non :

- la réduction de la taille du territoire assurant la nouvelle morphogenèse,

- l'arrêt de fonctionnement ou diapause du méristème,

- l'environnement écologique ou corrélatif auquel est soumis ce territoire.

Ils ont, en outre, attiré l'attention sur le fait que les cultures in vitro déclenchent, au fil des repiquages, dans le même temps, la miniaturisation du méristème édificateur de la plante et le retour vers une morphogenèse et des propriétés physiologiques de la jeune plante issue de la germination de la graine.

C'est dans le cadre de ces diverses acquisitions que nous avons essayé de situer les problèmes qui se posent à partir de la culture in vitro de l'artichaut, aux fins de multiplication conforme, et que nous avons tenté une approche biochimique de la question pour au moins un groupe de substances.

L'application de la culture in vitro à la multiplication accélérée de l'artichaut a fait l'objet de quelques recherches (ANCORA et al., 1979 ; MONCOUSIN, 1979, 1980a ; HARBAOUI \& DEBERGH, 1980). A la suite de ces travaux, des techniques de ramification intensive ont été développées et maîtrisées; elles conduisent pour la méthode que nous avons mise au point (MONCOUSIN, 1980 b) à une production de 3 à 3,510 boutures-rosettes, cn 6 mois, après 5 repiquages successifs.

Cependant, les boutures-rosettes ainsi obtenues ne manifestent qu'un faible taux d'enracinement limitant ainsi la portée pratique de ces résultats. C'est autour de cette caractéristique que nous avons cherché, par des essais d'analyse morphogénétique couplés à l'étude de l'évolution de l'activité peroxydasique des implants, à apporter notre contribution à la mise au point d'une méthode permettant de passer au niveau des applications agronomiques et à la mise en évidence du phénomène de rajeunissement acquis au cours des cultures in vitro.

En nous basant sur le comportement possible d'une bouture à la suite d'un traitement rhizogène, nous avons choisi 2 critères morphologiques - aptitude à la rhizogenèse, aptitude à la callogenèse - et un critère biochimique - l'évolution de l'activité peroxydasique des explants - qui a été utilisé avec succès pour apprécier l'influence des facteurs chimiques et physiques sur la rhizogenèse de l'artichaut (MONCOUSIN, 1981; MONCOUSIN \& GASPAR, $1983)$; de très bonnes corrélations existent, en effet, entre l'évolution de l'activité peroxydasique et l'aptitude à la rhizogenèse.

\section{MATÉRIEL ET MÉTHODES}

Les boutures-rosettes utilisécs pour les divers essais et comparaisons ont 2 origines possibles: il s'agit soit de jeunes plantes obtenues par semis en conditions aseptiques, soit d'explants, issus de divers niveaux (rangs) de repiquages successifs, élevés in vitro selon les techniques classiques (Moncousin, 1980b, 1981). Bien que la notion de cultivar ne soit pas particulièrement adaptée chez l'artichaut, nous avons essayé de réduire les risques de variations extérieurs à l'essai par l'emploi de graines et d'œilletons de «Vert de Laon».

Les principes généraux de préparations et d'utilisation du matériel végétal sont présentés dans la figure 1 mais nous préciserons cependant que :

- les graines sont installées sur le milieu de base (LINSMAIER \& SKOOG, 1965 : saccharose $28 \mathrm{~g} / \mathrm{l}$, agar $6 \mathrm{~g} / \mathrm{l}$ ) après désinfection à l'éthanol $(70 \mathrm{p} .100,20 \mathrm{mn})$ puis à l'hypochlorite de sodium $(14,5$ p. $100,20 \mathrm{mn})$ et rinçages à l'eau distillée stérile. La germination est réalisée à l'obscurité et à une température de $18^{\circ} \mathrm{C}$; dès ouverture des cotylédons, les tubes sont placés sous de nouvelles conditions (photopériode : $16 \mathrm{~h}$; lampes : Philips TL 33 ; éclairement : $100 \mu \mathrm{E} \mathrm{m}^{-2} \mathrm{~s}^{-1}$; température de jour : $25^{\circ} \mathrm{C}$; température de nuit : $20^{\circ} \mathrm{C}$ )

- les rosettes cultivées in vitro proviennent de la ramification intensive obtenue après installation des explants sur le milieu de prolifération (milieu de base + AIB $1 \mathrm{mg} / \mathrm{l}$, 2IP $10 \mathrm{mg} / \mathrm{l}$, L-tyrosine $60 \mathrm{mg} / \mathrm{l}$ ) dans des conditions d'environnement identiques à celles décrites précédemment. L'importance et la rapidité de la ramification permettent de disposer de plus de 70 boutures-rosettes, après 8 semaines de culture et seulement 2 repiquages successifs. Des fragments végétaux résultant de la division des touffes obtenues après $2,4,6,8,12$ et 15 repiquages successifs sont utilisés, au même moment, pour les divers essais.

Une semaine avant le début de l'expérience, les plantes de semis sont sorties de leur tube, la partie racinaire et les cotylédons sont supprimés. Les boutures ainsi obtenues ont 4 à 5 feuilles développées, longues de 5 à $7 \mathrm{~cm}$ pour les plus grandes. Au même moment, les touffes en ramification

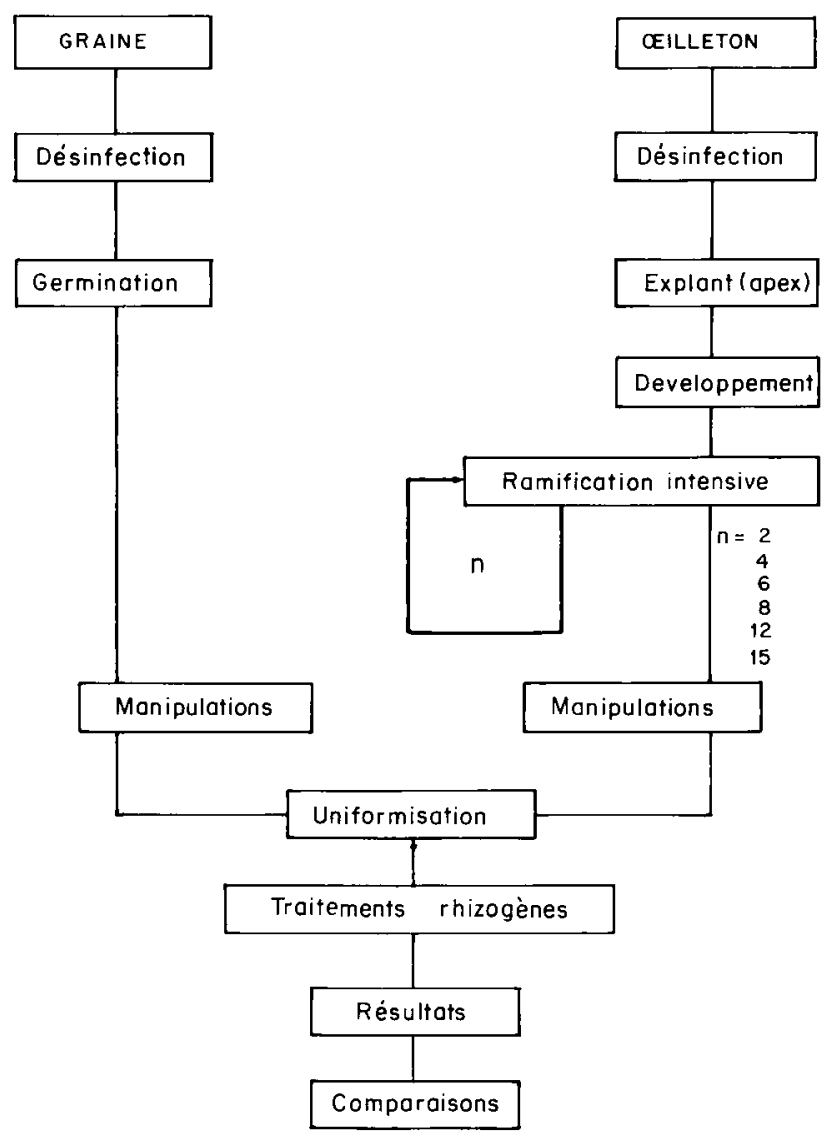

Figure 1

Protocole expérimental.

Experimental protocol. 
intensive sont divisées et les rosettes d'un développement proche de celui des plantes de semis sont retenues pour les essais. Toutes les boutures sont placées pendant une semaine sur un milieu simplifié (LinsmaIER \& SKOOG, 1965 ) dilué de moitié (saccharose $18 \mathrm{~g} / \mathrm{l}$, agar $6 \mathrm{~g} / \mathrm{l}$ ) pour homogénéiser le développement du matériel et pour éviter que les modifications du profil peroxydasique consécutives aux manipulations ne pertubent les réactions aux traitements rhizogènes.

Les traitements rhizogènes, expérimentés préalablement (MONCOUSIN, 1981 ; MONCOUSIN \& GASPAR, 1983), sont basés sur l'emploi de l'acide naphtalène acétique (ANA) et de l'ergocalciférol (vit. $\mathrm{D}_{2}$ ) et leurs effets comparés à ceux obtenus sur le milieu simplifié employé comme témoin. Les conditions d'environnement ne diffèrent des précédentes que par une diminution de l'éclairement $\left(60 \mu \mathrm{E} \mathrm{m}^{-2} \mathrm{~s}^{-1}\right)$.

Des travaux préalables (MONCOUSIN \& GASPAR, 1983) ayant montré que les mesures très précoces donnaient une bonne image des tendances rhizogènes, les racines adventives ont été dénombrées après $10 \mathrm{j}$ de traitement. Le comptage réalisé sous la loupe binoculaire ne prend en considération que les racines de plus de $1 \mathrm{~mm}$ de longueur. L'activité callogène est appréciée, visuellement, après $15 \mathrm{j}$ et seuls les implants produisant plus de $10 \mathrm{mg}$ de cals sont dénombrés. Les phénomènes morphologiques sont analysés sur 5 séries successives de 10 boutures par traitemınt (ANA $5 \mathrm{mg} / \mathrm{l}$, ANA $2 \mathrm{mg} / \mathrm{l}$, ANA $1 \mathrm{mg} / \mathrm{l}$, ANA $1 \mathrm{mg} / \mathrm{l}+$ vit. $\mathrm{D}_{2}$ $10 \mathrm{mg} / \mathrm{l}$, témoin) et par niveau de repiquages successifs. Un schéma identique est appliqué aux boutures de «semis».
L'activité peroxydasique journalière des boutures installées sur le milieu ANA + vit. $\mathrm{D}_{2}$ est déterminée par 3 séries de 5 mesures individuelles. La méthode, couramment utilisée (QUOIRIN et al., 1974 ; GASPAR \& VAN HOOF, 1976), est décrite par ailleurs (MONCOUSIN \& GASPAR, 1983).

Les tests statistiques, classiques, se résument à des analyses de variance simple, avec ou sans transformations préalables (DAGNÉLIE, 1975).

\section{RÉSULTATS}

Des différences appréciables du comportement morphogénétique apparaissent : elles peuvent être liées à la composition du milieu rhizogène ou au nombre de repiquages successifs.

Pour des raisons de clarté, nous avons dissocié les 2 possibilités :

\section{A. Phénomènes morphogénétiques liés à la composition du milieu}

L'évolution du pourcentage d'enracinement (tabl. 1) ne se fait pas de manière identique; des coefficients de variation fortement différents peuvent être calculés suivant les traitements. Ces coefficients sont particulièrement importants pour le traitement ANA $5 \mathrm{mg} / \mathrm{l}$ et pour le témoin; dans le $1^{\text {er }}$ cas, ils sont sans doute le reflet des 2 voies possibles pour la bouture (rhizogenèse ou calloge-

TABLEAU 1

Influence des traitements et du nombre de repiquages successifs. A. Evolution du pourcentage d'enracinement. B. Evolution du pourcentage d'implants callogènes. C. Evolution du nombre de racines formées.

Influence of treatments and of number of successive subcultures. A. Changes in rooting rate. B. Changes in the \% of callogenic explants. C. Changes in the number of roots formed.

\begin{tabular}{|c|c|c|c|c|c|c|}
\hline Niveaux & Traitements & $\begin{array}{l}\text { ANA } \\
5 \mathrm{mg} / 1\end{array}$ & $\begin{array}{l}\text { ANA } \\
2 \mathrm{mg} / 1\end{array}$ & $\begin{array}{l}\text { ANA } \\
1 \mathrm{mg} / 1\end{array}$ & $\begin{array}{l}\text { Zéro } \\
\mathrm{mg} / 1\end{array}$ & $\mathrm{ANA}+\mathrm{D}_{2}$ \\
\hline \multicolumn{7}{|l|}{ A } \\
\hline $\begin{array}{l}\text { Semis } \\
\text { Repiquage } \\
\text { Repiquage } \\
\text { Repiquage } \\
\text { Repiquage } \\
\text { Repiquage } \\
\text { Repiquage }\end{array}$ & $\begin{array}{r}15 \\
12 \\
8 \\
6 \\
4 \\
2\end{array}$ & $\begin{array}{r}4 \pm 2 \\
2 \pm 2 \\
4 \pm 2 \\
8 \pm 4 \\
24 \pm 5 \\
14 \pm 5 \\
4 \pm 4\end{array}$ & $\begin{array}{r}48 \pm 4 \\
50 \pm 7 \\
42 \pm 8 \\
40 \pm 7 \\
38 \pm 6 \\
34 \pm 5 \\
2 \pm 1\end{array}$ & $\begin{array}{r}54 \pm 5 \\
52 \pm 8 \\
48 \pm 4 \\
36 \pm 5 \\
22 \pm 4 \\
8 \pm 2 \\
2 \pm 1\end{array}$ & $\begin{array}{c}40 \pm 7 \\
24 \pm 8 \\
12 \pm 5 \\
10 \pm 7 \\
4 \pm 2 \\
0 \\
0\end{array}$ & $\begin{array}{c}68 \pm 8 \\
64 \pm 5 \\
54 \pm 5 \\
46 \pm 9 \\
24 \pm 5 \\
14 \pm 5 \\
0\end{array}$ \\
\hline \multicolumn{7}{|l|}{ B } \\
\hline $\begin{array}{l}\text { Semis } \\
\text { Repiquage } \\
\text { Repiquage } \\
\text { Repiquage } \\
\text { Repiquage } \\
\text { Repiquage } \\
\text { Repiquage }\end{array}$ & $\begin{array}{r}15 \\
12 \\
8 \\
6 \\
4 \\
2\end{array}$ & $\begin{array}{l}58 \pm 4 \\
58 \pm 8 \\
50 \pm 10 \\
22 \pm 11 \\
8 \pm 2 \\
4 \pm 2 \\
0\end{array}$ & $\begin{array}{c}48 \pm 8 \\
50 \pm 7 \\
40 \pm 7 \\
22 \pm 8 \\
6 \pm 4 \\
6 \pm 4 \\
0\end{array}$ & $\begin{array}{c}42 \pm 8 \\
40 \pm 10 \\
34 \pm 5 \\
4 \pm 4 \\
4 \pm 2 \\
0 \\
0\end{array}$ & $\begin{array}{l}6 \pm 4 \\
4 \pm 2 \\
2 \pm 2 \\
2 \pm 2 \\
0 \\
0 \\
0\end{array}$ & $\begin{array}{c}12 \pm 6 \\
12 \pm 7 \\
10 \pm 6 \\
6 \pm 4 \\
4 \pm 4 \\
2 \pm 2 \\
0\end{array}$ \\
\hline \\
\hline $\begin{array}{l}\text { Semis } \\
\text { Repiquage } \\
\text { Repiquage } \\
\text { Repiquage } \\
\text { Repiquage } \\
\text { Repiquage } \\
\text { Repiquage }\end{array}$ & $\begin{array}{r}15 \\
12 \\
8 \\
6 \\
4 \\
2\end{array}$ & $\begin{array}{l}0,04 \pm 0,03 \\
0,02 \pm 0,02 \\
0,04 \pm 0,03 \\
0,12 \pm 0,05 \\
0,36 \pm 0,09 \\
0,20 \pm 0,08 \\
0,04 \pm 0,03\end{array}$ & $\begin{array}{l}0,54 \pm 0,09 \\
0,52 \pm 0,08 \\
0,46 \pm 0,09 \\
0,86 \pm 0,15 \\
0,74 \pm 0,15 \\
0,44 \pm 0,10 \\
0,03 \pm 0,02\end{array}$ & $\begin{array}{l}0,82 \pm 0,14 \\
0,64 \pm 0,20 \\
0,78 \pm 0,13 \\
0,52 \pm 0,11 \\
0,26 \pm 0,07 \\
0,08 \pm 0,04 \\
0,02 \pm 0,02\end{array}$ & $\begin{array}{c}0,48 \pm 0,09 \\
0,26 \pm 0,07 \\
0,16 \pm 0,06 \\
0,12 \pm 0,06 \\
0,04 \pm 0,03 \\
0 \\
0\end{array}$ & $\begin{array}{c}1,02 \pm 0,15 \\
0,86 \pm 0,11 \\
0,68 \pm 0,10 \\
0,48 \pm 0,08 \\
0,30 \pm 0,08 \\
0,14 \pm 0,05 \\
0\end{array}$ \\
\hline
\end{tabular}


nèse) alors que dans le $2^{\mathrm{e}}$, ils sont le reflet d'une importante hétérogénéité physiologique consécutive aux repiquages successifs. Les analyses de variance révèlent que les essais sont hautement significatifs $(p=0,01)$ quels que soient les traitements envisagés. Le classement sur base de l'activité rhizogène (tabl. 2) met en évidence des réponses très différentes, à un même traitement, pour des boutures issues de niveaux de repiquages différents. Pour le témoin et pour les traitements à faible concentration hormonale ANA 2 et $1 \mathrm{mg} / \mathrm{l}$, les boutures issues des niveaux de repiquage 15 et 12 ont l'activité rhizogène la plus intense. Sous l'effet de doses plus élevées d'ANA $(5 \mathrm{mg} / \mathrm{l})$, ce sont les niveaux de repiquages intermédiaires $(6,4,8)$ qui ont les meilleurs pourcentages d'enracinement. Les boutures de "semis" se comportent de manière comparable à celles des niveaux de repiquages 12 et 15 . Le traitement $\mathrm{ANA}+\mathrm{D}_{2}$ est, à dose d'auxine identique, nettement plus rhizogène mais le comportement des divers types de boutures n'est nullement modifié par l'emploi de la vitamine $\mathrm{D}_{2}$.

L'activité callogène des boutures (tabl. 1) dépend elle aussi des traitements et des types de boutures installées. L'image obtenue dans les tableaux 1 et 2 est pratiquement inverse de celle obtenue dans l'appréciation du pouvoir rhizogène ; les traitements les plus riches en auxine sont les plus actifs. Les analyses de variance ne donnent des réponses significatives que pour les traitements auxiniques; le traitement témoin et le traitement $\mathrm{ANA}+\mathrm{D}_{2}$ ne sont pas significatifs.

Cependant, quel que soit le traitement envisagé, lc classement (tabl. 2) est toujours dominé par les boutures de « semis » ou par celles issues des repiquages successifs 12 et 15. L'adjonction de vitamine $D_{2}$ à la dose d'acide naphtalène acétique diminue sensiblement l'activité callogène des boutures.

La dernière comparaison basée sur le nombre de racines formées par implant permet de dégager des résultats identiques à ceux obtenus lors de la comparaison des activités rhizogènes des boutures (tabl. 1 et 2 ).

\section{B. Phénomènes morphogénétiques liés au niveau de repi- quage des boutures}

Le regroupement des divers résultats (tabl. 3) permet de noter, dès le premier abord, des similitudes de comportement entre les boutures de "semis" et celles issues des repiquages successifs les plus élevés (12 et 15). La dose

TABLEAU 2

Classement du nombre de repiquages en fonction des différents traitements: A. Activité rhizogène, B. Activité callogène, $C$. Nombre moyen de racines formées.

Les traits verticaux relient les résultats qui ne different pas statistiquement au niveau de probabilité choisi pour les tests ANOVA.

Ranking of number of subcultures following various treatments: A. Rhizogenic activity, B. Callogenic activity, C. Number of roots formed. Vertical lines link results which did not differ statistically at the probability level chosen for ANOVA tests.

\begin{tabular}{|c|c|c|c|c|c|}
\hline \multicolumn{5}{|c|}{ Traitements } & \multirow[b]{2}{*}{ ANOVA } \\
\hline Témoin & $\begin{array}{l}\text { ANA } \\
1 \mathrm{mg} / \mathrm{l}\end{array}$ & $\begin{array}{l}\text { ANA } \\
2 \mathrm{mg} / \mathrm{l}\end{array}$ & $\begin{array}{l}\text { ANA } \\
5 \mathrm{mg} / 1\end{array}$ & $\begin{array}{l}\text { ANA } \\
+D_{2}\end{array}$ & \\
\hline
\end{tabular}

A

\begin{tabular}{|c|c|c|c|c|c|c|}
\hline S & $\mathrm{S}$ & 15 & 6 & $\mathrm{~S}$ & & \\
\hline 15 & 15 & $\mathrm{~S}$ & 4 & 15 & ANA $5 \mathrm{mg} / 1$ & $F=6,43>F 0,01$ \\
\hline 12 & 12 & 12 & 8 & 12 & ANA $2 \mathrm{mg} / 1$ & $F=69,50>F_{0,01}$ \\
\hline 8 & 8 & 8 & $\mathrm{~S}$ & 8 & ANA $1 \mathrm{mg} / 1$ & $F=55,83>F_{0,01}$ \\
\hline 6 & 6 & 6 & 2 & 6 & Témoin & $\mathrm{F}=31,71>\mathrm{F}_{0,01}$ \\
\hline 4 & 4 & 4 & 12 & 4 & $\mathrm{ANA}+\mathrm{D}_{2}$ & $F=99,56>F_{0} 0,01$ \\
\hline 2 & 2 & 2 & 15 & 2 & & \\
\hline
\end{tabular}

B

\begin{tabular}{l|l|l|l|l|ll}
$\mathrm{S}$ & $\mathrm{S}$ & 15 & 15 & $\mathrm{~S}$ & & \\
15 & 15 & $\mathrm{~S}$ & $\mathrm{~S}$ & 15 & ANA $5 \mathrm{mg} / \mathrm{F}=43,70>\mathrm{F} 0,01$ \\
12 & 12 & 12 & 12 & 12 & ANA $2 \mathrm{mg} / 1$ & $\mathrm{~F}=40,47>\mathrm{F} 0,01$ \\
8 & 6 & 8 & 8 & 8 & ANA 1 mg/l & $\mathrm{F}=40,30>\mathrm{F} 0,01$ \\
6 & 8 & 6 & 6 & 6 & Témoin & $\mathrm{F}=0,43<\mathrm{F} 0,05$ \\
4 & 4 & 4 & 4 & 4 & ANA $+\mathrm{D}_{2}$ & $\mathrm{~F}=0,91<\mathrm{F} 0,05$ \\
2 & 2 & 2 & 2 & &
\end{tabular}

C

\begin{tabular}{|c|c|c|c|c|c|c|}
\hline $\mathrm{S}$ & 12 & 8 & 6 & $\mathrm{~S} \mid$ & & \\
\hline 15 & $\mathrm{~S}$ & 6 & 4 & 15 & ANA $5 \mathrm{mg} / \mathrm{l}$ & $F=5,37>F 0,01$ \\
\hline 12 | & 15 | & sl & 8 & 12 & ANA $2 \mathrm{mg} / \mathrm{l}$ & $F=6,65>F 0,01$ \\
\hline 8 & 8 & 15 & 2 & 81 & ANA $1 \mathrm{mg} / 1$ & $\mathrm{~F}=10,77>\mathrm{F} 0,01$ \\
\hline 6 & 6 & 12 & 12 & 6 & Témoin & $\mathrm{F}=9,84>\mathrm{F} 0,01$ \\
\hline 4 & 4 & 4 & $S$ & 4 & $\mathrm{ANA}+\mathrm{D}_{2}$ & $F=17,38>F 0,01$ \\
\hline 2 & 2 & 2 & 15 & 2 & & \\
\hline
\end{tabular}




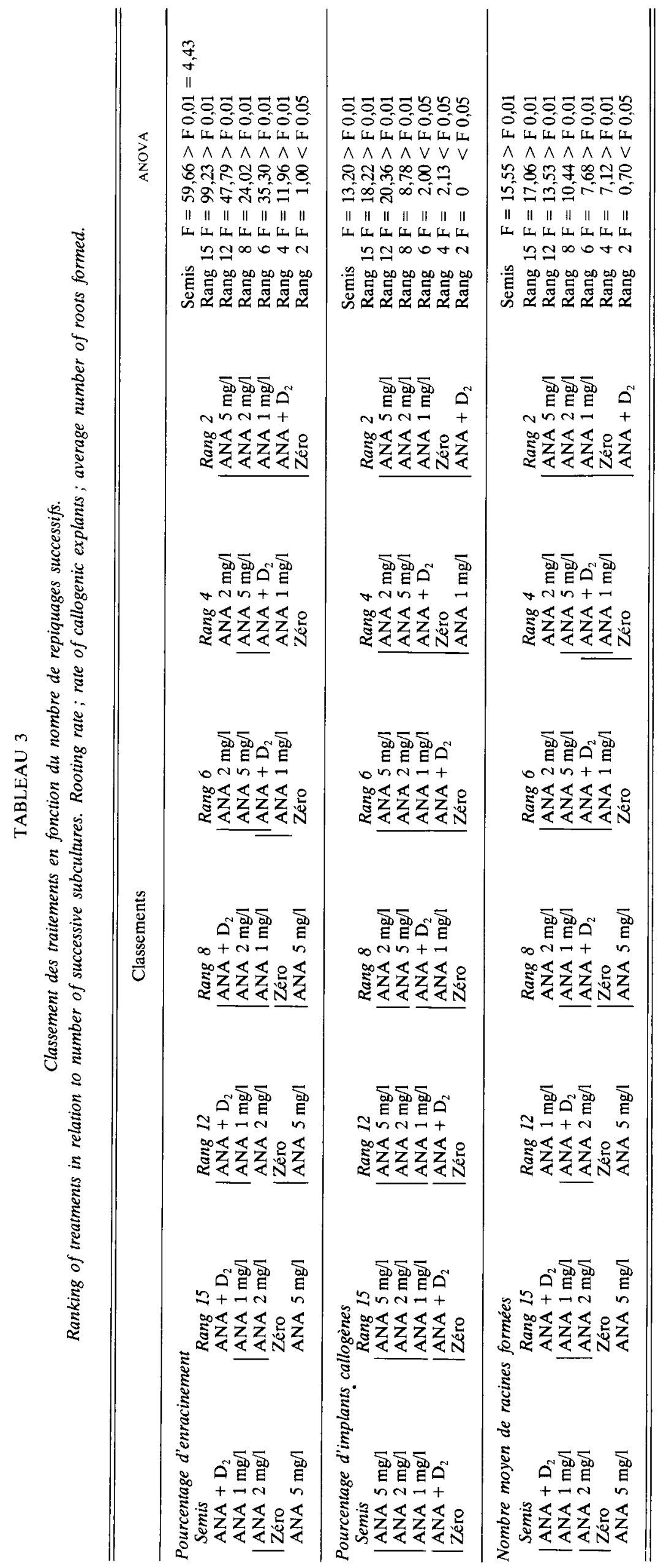


d'auxine nécessaire pour déclencher l'activité rhizogène décroît visiblement au fur et à mesure que le nombre de repiquages successifs augmente.

Une dose d'ANA trop élevée compromet l'activité rhizogène des boutures issues des niveaux 12 et 15 en favorisant une activité callogène intense.

Les tests statistiques permettent de rejeter l'hypothèse nulle à une probabilité $\mathrm{p}=0,01$, dans la majorité des cas ; par contre, seules les comparaisons des résultats obtenus avec les boutures de repiquages 2 sont significativement différentes $(p=0,05)$ et ceci pour les 3 comportements morphogénétiques choisis.

\section{Evolution de l'activité peroxydasique (fig. 2)}

Quel que soit le type de boutures installé sur le milieu ANA + vit $D_{2}$, l'activité peroxydasique, expriméc par le rapport des poids de peroxydases et de protéines, varie pendant les phases supposées d'induction et d'initiation racinaire, mais les évolutions diffèrent les unes des autres soit sur le plan de l'intensité, soit sur le plan de la durée des 2 phases, soit sur les 2 plans. Les boutures des repiquages 2 , 4 ou 6 ne montrent que de faibles variations d'activité peroxydasique ; celles du $8^{\mathrm{e}}$ repiquage ont une activité en augmentation plus rapide $(250,77$ après $5 \mathrm{j})$ qui décroît ensuite régulièrement. Seules les boutures issues des repiquages 12 et 15 ont des activités qui évoluent parallèlement à l'activité peroxydasique des boutures juvéniles (semis) ; la montée est aussi rapide ( 4 j) et les activités maximales sont très proches ísemis : $272,38 \pm 11,41$; repiquage 15 : $254,71 \pm 13,75$; repiquage $12: 245,41 \pm 7,73$ ).

La diminution d'activité peroxydasique se fait progressivement pour tous les types de boutures, tout au plus faut-il noter qu'elle est plus nette pendant les 2 premicrs jours pour les boutures juvéniles et pour celles des repiquages 12 , 15.

\section{DISCUSSION ET CONCLUSIONS}

Les réponses aux tests rhizogènes (pourcentage de boutures enracinées, nombre de racines formées) sont étroitement liées au nombre de repiquages successifs ayant permis d'obtenir les boutures. Les résultats sont d'autant plus probants que ces repiquages ont été nombreux ; après 12 ou 15 repiquages, les boutures ont acquis un comportement et des aptitudes morphogénétiques comparables à ceux des boutures issues de semis et considérées, à juste titre, comme juvéniles. Ces modifications d'aptitude rappellent celles apparues lors des multiplications intensives in vitro de Citrus (BOUzID \& LASRAM, 1971), de Pinus (FRANClet et al., 1980), de Vitis (Favre, 1970, 1973) et de Dahlia (WATELET-GONOD \& FAVRE, 1981). Tout se passe comme si les repiquages successifs permettaient un retour en arrière du végétal et ceci qu'il soit ligneux ou herbacé

Il faut signaler un comportement morphologique particulier qui n'a pasi ćté quantifié : dès le début de la phase d'initiation racinaire, les nouvelles feuilles formécs in vitro ont une morphologie différente de celles formées précédemment (fcuilles gris verdâtre, velues, indentées au lieu de feuilles vert clair, glabres et entières) et ceci sans qu'il y ait modification des conditions écologiques ou nutritives.

Le pourcentage de boutures ayant des réactions callogènes est lié, lui aussi, au nombre de repiquages subis. Les rosettes foliaires des repiquages $8,12,15$ se sont toujours montrées plus aptes à la callogenèse, mais leurs réactions sont d'autant plus importantes que le milicu est plus riche en

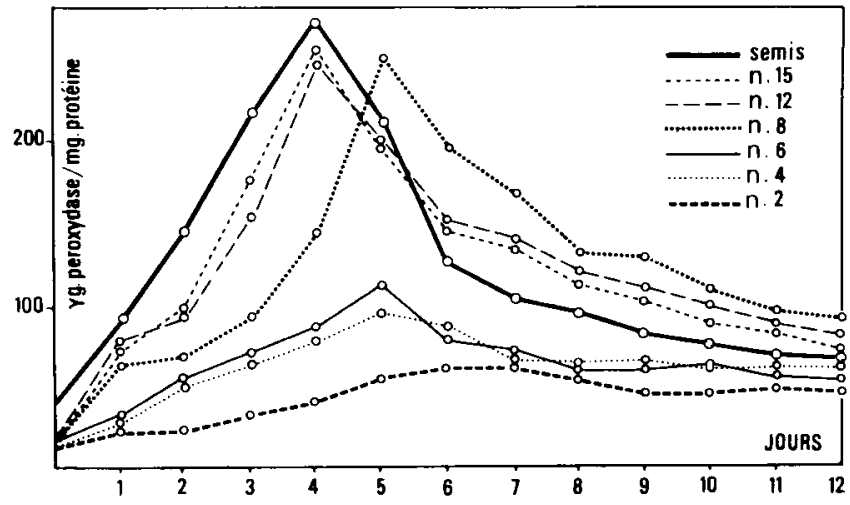

Figure 2

Evolution de l'activité peroxydasique des boutures-rosettes en fonction du temps et du nombre de repiquages successifs (milieu $A N A+$ ergocalciférol).

Changes in peroxidase activity of rosette cuttings with time and number of successive subcultures (ANA medium + ergocalciferol).

auxine. Les boutures juvéniles ont un comportement très orienté vers la callogenèse ainsi que l'avaient déjà montré DE LEO \& GRECO (1976).

L'utilisation du milieu ANA + vit. $\mathrm{D}_{2}$ comme inducteur de rhizogenèse se révèle bénéfique pour tous les types de boutures. Les activités rhizogènes sont toujours supérieures alors que la propension à former des cals diminue; ces particularités augmentent, par ailleurs, très fortement le pourcentage de réussite lors du retour en conditions septiques.

Les racines formées après traitement $\mathrm{ANA}+$ vit. $\mathrm{D}_{2}$ sont plus longues, plus nacrécs que celles obtenues après un traitement à dose identique d'ANA mais sans ergocalciférol.

L'utilisation de l'évolution de l'activité peroxydasique, pendant un traitement rhizogène, comme marqueur biochimique des potentialités réacquises pendant la multiplication in vitro est particulièrement adéquate. Non seulement des différences nettes apparaissent entre les activités des boutures des divers repiquages mais l'évolution de l'activité peroxydasique des boutures issues des repiquages 12,15 se rapproche de celle des boutures juvéniles. La comparaison des réactions au traitement $\mathrm{ANA}+\mathrm{D}_{2}$ (tabl. 1, fig. 2) met en évidence une corrélation étroite entre la montée de l'activité peroxydasique (temps et intensité) et les activités rhizogènes. Dans les 3 cas, les tests statistiques sont hautement significatifs et il n'existe pas de différence significative $(\mathrm{p}=0,01)$ entre les réponses des boutures juvéniles et celles du repiquage 15 . Les résultats obtenus avec les boutures du repiquage 12 ne diffèrent à la même probabilité de ccux du repiquage 15 que dans la comparaison des pourcentages d'enracinement (tabl. 4).

L'évolution de l'activité peroxydasique est le reflet d'une réaction plus ou moins intense à un traitement rhizogène car les mesures sont réalisćes sur des poids identiques de limbe foliaire et font intervenir le rapport entre le poids de peroxydases et le poids de protéines.

La nécessité d'une augmentation rapide de l'activité peroxydasique semble aussi déterminante que l'importance du pic pour l'obtention de caractères rhizogènes marqués. Cette constatation avait, d'ailleurs, été faite précédemment, en comparant les effets de diverses substances sur la rhizogenèse d'explants du rang $n^{\circ} 8$ (MONCOUSIN \& GASPAR, 1983). L'utilisation du test d'activité peroxydasique 


\section{TABLEAU 4}

Résultats de l'activité peroxydasique, nombre de racines et pourcentages d'enracinement.

Results on peroxidase activity, number of roots and rooting rate.

\begin{tabular}{|c|c|c|c|c|}
\hline \multirow{2}{*}{ Rangs } & \multirow{2}{*}{$\begin{array}{l}\text { Rapport } \\
\text { peroxydasique } \\
\text { maximal }\end{array}$} & \multirow{2}{*}{ Jours } & \multicolumn{2}{|c|}{ Enracinement } \\
\hline & & & Nombre de racines & Pourcentage \\
\hline Semis & 272,38 & 4 & $1,02 \pm 0,15$ & $68 \pm 81$ \\
\hline Rang 15 & 254,71 & 4 & $0,86 \pm 0,11$ & $64 \pm 51$ \\
\hline Rang 12 & 245,41 & 4 & $0,68 \pm 0,10$ & $54 \pm 5$ \\
\hline Rang 8 & 250,77 & 5 & $0,48 \pm 0,08$ & $46 \pm 91$ \\
\hline Rang 6 & 110,61 & 5 & $0,30 \pm 0,08$ & $24 \pm 5$ \\
\hline Rang 4 & 94,59 & 5 & $0,14 \pm 0,05$ & $14 \pm 5$ \\
\hline Rang 2 & 68,39 & 7 & 0 & 0 \\
\hline
\end{tabular}

permet de surmonter les problèmes délicats de l'enracinement rencontrés chez Cynara scolymus L., en facilitant le choix des niveaux de repiquages et des substances les plus aptes à provoquer la rhizogenèse, mais aussi, de trouver un marqueur biochimique quantifiable de la réacquisition, au fil des repiquages, de propriétés du type de celles de jeune plante issue de graine.

Outre leur intérêt fondamental, l'ensemble des faits que nous avons mis en évidence, au moins avec $C$. scolymus, devrait permettre de mieux maîtriser les conditions de milieu dans le cadre de l'utilisation in vitro des plantes d'intérêt agronomique.

Reçu le 16 décembre 1982. Accepté le 8 septembre 1983.

\section{REMERCIEMENTS}

Nous tenons à remercier MM. les Professcurs GASPAR et NOzERAN pour leur appui et leurs conseils pendant la réalisation de ce travail.

\section{RÉFÉRENCES BIBLIOGRAPHIQUES}

Ancora G., Belli-Donini M., Cuozzo L., 1979. Moltiplicazione in vitro di apici vegetativi di carciofo. Atti $3^{e}$ Congr. Int. Carciofo, Bari ; Tip. Laterza, Bari, 239.

Bouzid S., Lasram M., 1971. Utilisation de cultures in vitro pour l'obtention de clones de Citrus homogènes et de bon état sanitairc. $8^{e}$ Congr. Int. Agrumic. Mediterr. "Holland Pearl" C.L.A.M. (Madrid), V. II, 1.

Dagnelie P., 1975. Théorie et méthodes statistiques. Presses agronomiques. Duculot, Gembloux.

De Leo P., Greco B., 1976. Nuova tecnica di propagazione del carciofo coltura in vitro di meristemi apicali. Nuovi studi sul carciofo, Bari, 1976, Minerva Medica ed., 657.

Doorenbos J., 1953. Rejuvenation of Hedera helix in graft combination Preb. 115, Wageningen, nov. 1953, 99.

Doorenbos J., 1965. Juvenile and adult phases in woody plants. Handb. Pflanzenphysiol., 15 (1), 1222.

Favre J.M., 1970. Premiers résultats concernant lobtention in vitro de néoformations caulinaires chez la vigne. Ann. Amél. Plant., 27, 151.

Favre J.M., 1973. Effets corrélatifs de facteurs internes et externes sur la rhizogenèse d'un clone de vigne (Vitis riparia $\times$ Vitis rupestris) cultivé in vitro. Rev. Gén. Bot., 80, 279.

Franclet A., 1969. Vers une production en masse de cyprès de forme contrôlée. Proceed. 2nd FAOIIUFRO World. Consult. For. Tree Breeding, Washington FO/FTB 69, 11.

Franclet A., David A., David H., Boulay M., 1980. Première mise en évidence morphologique d'un rajeunissement de méristèmes caulinaircs de pin maritime âgé (Pinus pinaster Sol.) C.R.Acad. Sci. Paris, 290, 297.

Gaspar T., Van Hoof P., 1976. Application d'un test peroxydasique dans le choix des plantes d'asperge à propager in vitro. Rev. Agricult. 3, 583 .

Gregory L.E., 1951. Una nota sobre el enraizamiento de los clones de Hevea. Turrialba, 1, 201.

Harbaoui Y., Debergh P., 1980. Multiplication in vitro de clones sélectionnés d'artichaut (Cynara scolymus L.). In Application de la culture in vitro à l'amélioration des plantes potagères Eucarpia, Versailles, 1.

Linsmaier E.M., Skoog F., 1965. Organic growth factor requirements of tobacco tissue cultures. Physiol. Plant., 18, 100.

Moncousin C., 1979. Multiplication végétative accélérée et sélection bactérienne de Cynara scolymus L. Atti $3^{e}$ Congr. Int. Carciofo, Bari ; Tip. Laterza, Bari, 219.

Moncousin C., 1980a. Multiplication végétative accélérée de Cynara scolymus L.: Résultats préliminaires. Rev. Hortic. Suisse, 53, 149.

Moncousin C., 1980b. Micropropagation in vitro de Cynara scolymus L.: Conséquences bactériologiques. In Application de la culture in vitro à l'amélioration des plantes potagères, Eucarpia. Versaillcs, 8 .

Moncousin C., 1981. Multiplication végétative accélérée de Cynara scolymus L.: Il. Amćlioration des diverses techniques. Rev. Hortic. Suisse, 54, 105.

Moncousin C., Gaspar T., 1983. Peroxidase as a marker for rooting improvement of Cynara scolymus L. cultured in vitro. Biochem. Physiol. Pflanz., 178, 263.

Nozeran R., 1978. Réflexions sur les enchaînements de fonctionnement au cours du cycle des végétaux supérieurs Bull. Soc. Bot. Fr., $125,263$.

Nozeran R., Bancilhon L., 1972. Les cultures in vitro en tant que technique pour l'approche de problèmes posés par l'amélioration des plantes. Ann. Amél. Plant., 22 (2), 167.

Nozeran R., Ducreux G., Rossignol-Bancilhon L., 1982. Réflexions sur les problèmes de rajeunissement chez les végétaux. Bull. Soc. Bot. Fr., 129, 107-130.

Quoirin M., Boxus P., Gaspar T., 1974. Root initiation and isoperoxidases of stem tip cuttings from mature Prunus plants. Physiol. Vég., 12, 165.

Watelet-Gonod M.C., Favre J.M., 1981. Miniaturisation ct rajeunissement chez Dahlia variabilis cv "Télévision", cultivé in vitro. Ann. Soc. Nat. Bot., $13(2-3), 51$. 